University of Nebraska - Lincoln

DigitalCommons@University of Nebraska - Lincoln

2010

\title{
Entropy localization in magnetic compounds and thin-film nanostructures
}

\author{
Ralph Skomski \\ University of Nebraska-Lincoln, rskomski2@unl.edu \\ Christian Binek \\ University of Nebraska-Lincoln, cbinek@unl.edu \\ Steven A. Michalski \\ University of Nebraska-Lincoln, smichalski2@unl.edu \\ Tathagata Mukherjee \\ University of Nebraska-Lincoln, tatha.muk@gmail.com \\ Axel Enders \\ University of Nebraska-Lincoln, a.enders@me.com \\ See next page for additional authors
}

Follow this and additional works at: https://digitalcommons.unl.edu/physicsskomski

Part of the Physics Commons

Skomski, Ralph; Binek, Christian; Michalski, Steven A.; Mukherjee, Tathagata; Enders, Axel; and Sellmyer, David J., "Entropy localization in magnetic compounds and thin-film nanostructures" (2010). Ralph Skomski Publications. 68.

https://digitalcommons.unl.edu/physicsskomski/68

This Article is brought to you for free and open access by the Research Papers in Physics and Astronomy at DigitalCommons@University of Nebraska - Lincoln. It has been accepted for inclusion in Ralph Skomski Publications by an authorized administrator of DigitalCommons@University of Nebraska - Lincoln. 


\section{Authors}

Ralph Skomski, Christian Binek, Steven A. Michalski, Tathagata Mukherjee, Axel Enders, and David J. Sellmyer 


\title{
Entropy localization in magnetic compounds and thin-film nanostructures
}

\author{
R. Skomski, ${ }^{\text {a) }}$ Ch. Binek, S. Michalski, T. Mukherjee, A. Enders, and D. J. Sellmyer \\ Department of Physics and Astronomy and Nebraska Center for Materials and Nanoscience, University of \\ Nebraska, Lincoln, Nebraska 68588, USA
}

(Presented 22 January 2010; received 31 October 2009; accepted 10 December 2009; published online 21 April 2010)

\begin{abstract}
The effect of nanostructuring on the magnetic entropy of materials for room-temperature magnetic cooling is investigated by model calculations. The materials are structurally inhomogeneous with a large number of nonequivalent crystallographic sites. In the mean-field Heisenberg model, the entropy density is a unique function of the local magnetization so that the coupled set of nonlinear mean-field equations yields not only the magnetization but also the entropy density. Since most of the entropy is localized near grain boundaries, nanomagnetic cooling requires small feature sizes. Magnetic anisotropy is a substantial complication, even on a mean-field level, but the corresponding corrections are often very small. @ 2010 American Institute of Physics. [doi:10.1063/1.3367960]
\end{abstract}

\section{INTRODUCTION}

Magnetic refrigeration, based on the change in isothermal magnetic entropy, is an important development in the quest for energy-efficient and environmentally friendly technologies. While some elements, especially $\mathrm{Gd}$, exhibit an appreciable magnetocaloric effect, there is a trend toward complex magnetic structures, such as ternary compounds, ${ }^{1,2}$ ultrathin films, ${ }^{3}$ and small-scale granular nanostructures. ${ }^{4,5}$ This structuring helps to improve the performance of the materials by maximizing the entropy change, suppressing hysteresis looses, adjusting the operation temperature, and reducing the required magnetic field.

Figure 1 illustrates the local spin structure involved in the cooling process. A magnetic field aligns the spin (left) and subsequent field removal causes the spins to disorder (right). Microscopically, the spin disorder is realized by the interaction with a heat bath (random thermal motion of atoms). For isolated atoms (paramagnetic ions), the effect is limited to low temperatures because the temperature equivalent of the Zeeman energy $-g J \mu_{\mathrm{B}} \mu_{\mathrm{o}} H$ is only about $1 \mathrm{~K}$ for typical fields of order $\mu_{0} H=1$ T. This means that typical laboratory-scale magnetic fields are not able to realize roomtemperature saturation. Alternatively, huge magnetic fields (around $100 \mathrm{~T}$ ) are necessary to operate such a system at temperatures of technological interest.

The are two basic ways to increase the operation temperature. The traditional way is to use first- or second-order phase transitions where the interatomic exchange $J_{\mathrm{ex}}$ enhances the effect of the magnetic field. ${ }^{1,2}$ In the vicinity of a second-order phase transition $\left(T \sim T_{\mathrm{c}}\right)$, the entropy is associated with critical fluctuations, which are suppressed by a magnetic field. First-order transitions help if they occur at a temperature where the involved phases have substantially different entropies. For example, a transition from a ferromagnetic (FM) to a Pauli-paramagnetic phase is not very helpful because both phases have low entropies. An addi-

${ }^{\text {a)} E l e c t r o n i c ~ m a i l: ~ r s k o m s k i @ n e b . r r . c o m . ~}$ tional consideration is structural and/or magnetic hysteresis which creates heat during thermodynamic cycling and is harmful in any system.

We plan to exploit phase-transition effects but our main interest is to use granular and thin-film nanostructures. ${ }^{4,5}$ Embedding FM nanoparticles, such as bcc Fe, fcc Co or $\mathrm{DO}_{3}$-ordered $\mathrm{Fe}_{3} \mathrm{Si}$, in a matrix corresponds to the use of large "macrospins" $J \sim N$, where $N$ is roughly equal to the number of atoms in the cluster or particle. The matrix consists of a magnetic material with an ordering temperature near room-temperature, for example, a Gd- or Mn-containing alloy.

The main effect of the nanostructuring is that $J \sim N$ reduces the magnetic field necessary for saturation $H \sim 1 / N$. The entropy $S$ increases with $N$, but this increase is only logarithmic and overcompensated by the large heat capacity $C \sim N$, and it is not possible to use big particles. In fact, even for noninteracting particles, ${ }^{4} N=300$ is sufficient to ensure a substantial spin polarization in a field of $1 \mathrm{~T}^{6}$ A related point is the effective range of the exchange interactions. Exchange-coupled nanocomposites containing a phase with a high Curie temperature $\left(T_{\mathrm{H}}\right)$ and a phase with a low Curie temperature $\left(T_{\mathrm{L}}\right)$ have a single Curie temperature $T_{\mathrm{c}} \sim T_{\mathrm{H}} \cdot{ }^{7,8}$ However, between $T_{\mathrm{L}}$ and $T_{\mathrm{c}}$, the spontaneous magnetization decays exponentially in the $T_{\mathrm{L}}$ phase, and a similar behavior may be expected for the entropy density $\eta=\mathrm{d} S / \mathrm{d} V$.

In this paper, we investigate how the entropy density is affected by the nanostructuring. We use an isotropic mean-
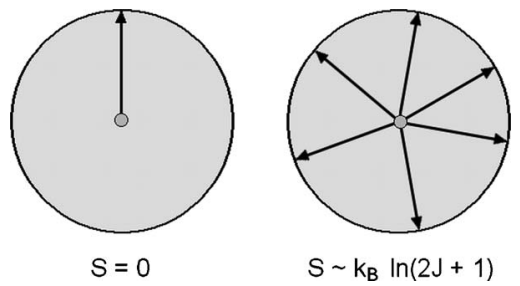

FIG. 1. Entropy change caused by the removal of an external magnetic field. The enhancement of the entropy $S$, visualized by snapshots of an atomic spin at different times (right), requires heat (TS), and this heat is taken from the environment that surrounds the spin. 

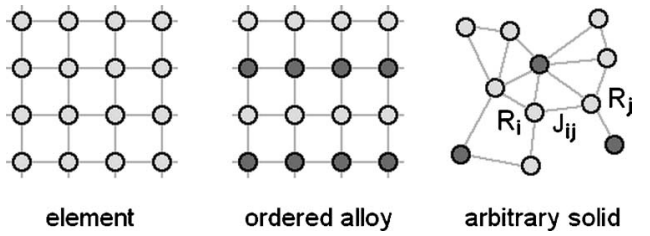

FIG. 2. Atomic entropy contributions. The interatomic exchange determines the local magnetization and, indirectly, the entropy contribution of each atom. The case of arbitrary solids includes magnetic multilayers and granular magnetic nanostructures.

field model to investigate $\eta(\mathbf{r})$ near interfaces and discuss how the entropy density is affected by magnetic anisotropy and by critical fluctuations.

\section{MODEL AND RESULTS}

The structures are modeled by considering many crystallographically nonequivalent sites (index $i$ ) with different thermally averaged magnetizations $m_{\mathrm{i}}=\left\langle s_{\mathrm{i}}\right\rangle$ or $m_{\mathrm{i}}=\left\langle\mu_{\mathrm{i}}\right\rangle / \mu_{\mathrm{is}}$. For example, the local magnetization $m_{\mathrm{i}}$ in a particle depends on the distance from the surface. ${ }^{9}$ This leads to a spatial distribution of the entropy density $\eta(\mathbf{r}) \sim S\left(\mathbf{R}_{\mathrm{i}}\right)=S_{\mathrm{i}}$ whose sum or integral enters relations such as $(\partial S / \partial H)_{\mathrm{T}}$ $=(\partial M / \partial T)_{\mathrm{H}}$. The mean-field partition function $Z(H / T)$ yields the local entropy $S_{\mathrm{i}}$ as a unique function of the average local magnetization $m(\mathbf{r})$

$$
\left\langle m_{\mathrm{i}}\right\rangle=B(i)\left(\frac{h_{\mathrm{i}}+\sum_{\mathrm{j}} J_{\mathrm{ij}}\left\langle m_{\mathrm{j}}\right\rangle}{k_{\mathrm{B}} T}\right),
$$

where $B(i)$ is the Brillouin function of the $i$ th atom and $h_{\mathrm{i}}-\mu_{\mathrm{o}} \mu_{\mathrm{i}} H$. [Here we use $B(i)$ rather than $B_{\mathrm{J}}$ or $B_{\mathrm{S}}$ to avoid confusion with the entropy $S$ and the exchange $J]$. Each nonequivalent site contributes one nonlinear equation, as illustrated in Fig. 2, and the resulting temperature dependence of the magnetization curves may differ qualitatively from the predictions of the homogeneous mean-field theory. ${ }^{7}$

In terms of inverse Brillouin functions $B(i)^{-1}$, the entropy per atom is

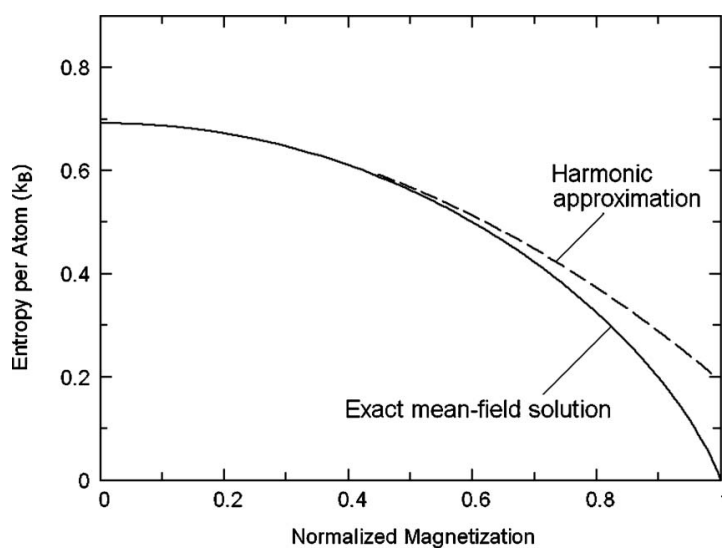

FIG. 3. Entropy density as a function of the local magnetization. The solid curve is based on the mean-field solution of the spin-1/2 Ising model. The dashed line shows the harmonic approximation $S_{\mathrm{i}}=k_{\mathrm{B}}\left[\ln (2)-m_{\mathrm{i}}^{2} / 2\right]$.

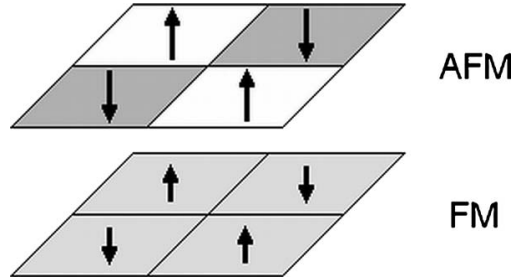

FIG. 4. Schematic spin structure at the ordering temperature. The phase with the higher ordering temperature (here AFM) imposes its spin structure and entropy density onto the FM phase at the bottom.

$$
S_{\mathrm{i}}=k_{\mathrm{B}} \ln Z\left[B(i)^{-1}\left(m_{\mathrm{i}}\right)\right]-k_{\mathrm{B}} m_{\mathrm{i}} B(i)^{-1}\left(m_{\mathrm{i}}\right) .
$$

This function decreases with increasing $m_{\mathrm{i}}$ from $k_{\mathrm{B}} \ln \left(2 J_{\mathrm{i}}\right.$ $+1)$ at $m_{\mathrm{i}}=0$ to 0 as $m_{\mathrm{i}}$ approaches saturation. In the spin-1/2 Ising model, $B_{\mathrm{J}}^{-1}(m)=\operatorname{atanh}(m), S(T=0)=k_{\mathrm{B}} \ln (2)$, and, for small local magnetization $m_{\mathrm{i}}, S_{\mathrm{i}}=k_{\mathrm{B}}\left(\ln (2)-m_{\mathrm{i}}^{2} / 2\right)$. Figure 3 illustrates the dependence of $S$ on $m$ for this spin-1/2 Ising case. These equations mean that the picture of Fig. 1 can be generalized to complicated alloys and nanostructures.

Equation (2) reduces the problem of entropy determination to the simpler problem ${ }^{7,8,10,11}$ of finding the local magnetization $m_{\mathrm{i}}$ as a function of $H$ and $T$. For example, composite thin films such as $\mathrm{Cr} / \mathrm{Co}{ }^{3}$ each layer has a different magnetization $m_{\mathrm{i}}$, and the individual layer magnetizations exhibit an additional lateral modulation due to the anti-FM (AFM) interactions of the Cr. The entropy density is determined by solving the system's coupled mean-field equations. It rapidly decreases from the interface, ${ }^{7,9}$ and the decrease is described by $\exp (-2 z / R)$, where $R \sim\left|1-T / T_{\mathrm{c}}\right|^{-\nu}$ is the correlation or localization length in the corresponding phase.

Figure 4 shows local magnetization for a simple FMAFM thin-film structure. The model film has four nonequivalent sites, two in each layer, so that Eq. (1) reduces to a set of four nonlinear equations. In the case shown in Fig. 4, the AFM interactions are sufficiently strong to yield an AFM spin polarization of the FM phase. The spin structure and the entropy depend on the involved inter- and intraplane exchange interactions. In Fig. 4, the entropy does not show any unusual features but the interactions can be used to move the operating temperature into a convenient range, as in the Cr/Co system. ${ }^{3}$

In other cases, the interatomic coupling strongly suppresses the magnetic cooling. Figure 5 shows the spin structure of a isotropic two-sublattice antiferromagnet $(i=1,2)$

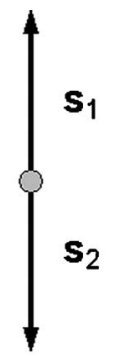

(a)

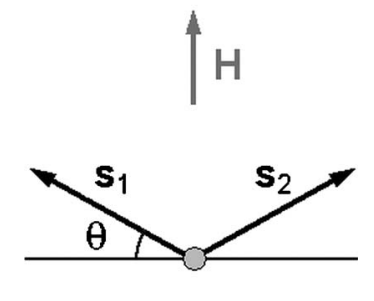

(b)
FIG. 5. Isotropic antiferromagnet with two sublattices $i=1,2$ in (a) zero field and (b) nonzero field. A small field causes the magnetization to switch by about $90^{\circ}$ (b). 
with negative intersublattice and zero intrasublattice exchange in a weak magnetic field. The magnetization angle $\theta$, defined in Fig. 5, depends on the ratio of field to exchange but is independent of temperature. This means that $\chi$ =const. below $T_{\mathrm{N}}$ (Ref. 10) and $(\partial M / \partial T)_{\mathrm{H}}=\partial S / \partial H=0$. Consequently, the magnetic field affects the internal energy (exchange) but leaves the entropy unchanged.

\section{DISCUSSION AND CONCLUSIONS}

Entropy expressions similar to the above-discussed equations have long been used to derive and discuss equations of state for homogeneous magnets, ${ }^{10}$ but the local character of $S$ and the unique relation between $S$ and $m$ are nontrivial. The local character of the entropy is a mean-field property whereas the uniqueness reflects the isotropic character of the model. Magnetic anisotropy has a huge impact on macroscopic magnetic properties from the ordering temperature $^{12}$ to hysteresis and coercivity. However, on an atomic scale, the magnetic anisotropy and its contribution to the mean-field entropy are small. This is because the temperature equivalents of the anisotropy energy per atom, about $1 \mathrm{mK}$ for soft-magnetic materials and about $1 \mathrm{~K}$ for hardmagnetic materials, $7,13,14$ are much smaller than typical ordering temperatures of several $100 \mathrm{~K}$. There are cases where anisotropy and exchange energies are comparable, such as some rare-earth magnets ${ }^{15}$ and surface alloys, ${ }^{16}$ but these materials have very low Curie temperatures and are not suitable for interest in room-temperature magnetic refrigeration.

Near a second-order phase transition, the long-range character of the critical fluctuations means that the entropy density is no longer a function of the local magnetization. However, as in the mean-field case, the corresponding correlation length scales as $a\left|1-T / T_{\mathrm{c}}\right|^{-\nu}$, where a is the interatomic distance and $T_{\mathrm{c}}(\mathbf{r}) \sim z J$ is the "local" Curie temperature. ${ }^{7}$ Unless the involved phases have very similar Curie tempera- tures, the thermodynamic correlations decay very rapidly in each phase and Eq. (1) is a good approximation for nanostructured magnets.

In conclusion, our mean-field analysis shows that nanostructuring affects the entropy density of magnetocaloric materials via the local magnetization, mirroring the generally inhomogeneous local magnetization. The anisotropy has a great impact on the macroscopic properties, including magnetic ordering, but on a local scale, it is often a small correction to the leading isotropic entropy contribution.

\section{ACKNOWLEDGMENTS}

This work has been supported by NRI, NSF-MRSEC (DMR 0213808), and NCMN.

${ }^{1}$ E. Brück, O. Tegus, D. T. C. Thanh, and K. H. J. Buschow, J. Magn. Magn. Mater. 310, 2793 (2007).

${ }^{2}$ K. A. Gschneidner, Jr., V. K. Pecharsky, and A. O. Tsokol, Rep. Prog. Phys. 68, 1479 (2005).

${ }^{3}$ T. Mukherjee, S. Sahoo, R. Skomski, D. J. Sellmyer, and Ch. Binek, Phys. Rev. B 79, 144406 (2009).

${ }^{4}$ R. D. McMichael, R. D. Shull, L. J. Swartzendruber, and L. H. Bennett, J. Magn. Magn. Mater. 111, 29 (1992).

${ }^{5}$ R. Skomski, Ch. Binek, T. Mukherjee, S. Sahoo, and D. J. Sellmyer, J. Appl. Phys. 103, 07B329 (2008).

${ }^{6}$ As a rule of thumb, $1 \mathrm{~nm}^{3}$ corresponds to $\mathrm{N}=100$.

${ }^{7}$ R. Skomski and D. J. Sellmyer, J. Appl. Phys. 87, 4756 (2000).

${ }^{8}$ R. Skomski, Simple Models of Magnetism (University Press, Oxford, 2008).

${ }^{9}$ R. Skomski, C. Waldfried, and P. A. Dowben, J. Phys.: Condens. Matter 10, 5833 (1998).

${ }^{10}$ J. S. Smart, Effective Field Theories of Magnetism (Saunders, Philadephia, 1966).

${ }^{11}$ C. Binek, Ising-Type Antiferromagnets (Springer, Berlin, 2003).

${ }^{12}$ M. Bander and D. L. Mills, Phys. Rev. B 38, 12015 (1988).

${ }^{13}$ R. Skomski, J. Appl. Phys. 83, 6724 (1998).

${ }^{14}$ R. Skomski and J. M. D. Coey, Permanent Magnetism (IOP, Bristol, 1999).

${ }^{15}$ D. J. Sellmyer and S. Nafis, Phys. Rev. Lett. 57, 1173 (1986).

${ }^{16}$ J. Honolka, T. Y. Lee, K. Kuhnke, A. Enders, R. Skomski, S. Bornemann, S. Mankovsky, J. Minár, J. Staunton, H. Ebert, M. Hessler, K. Fauth, G. Schütz, A. Buchsbaum, M. Schmid, P. Varga, and K. Kern, Phys. Rev. Lett. 102, 067207 (2009). 